\title{
COMPTEL OBSERVATIONS OF AGNs
}

\author{
H. Steinle, ${ }^{*}$ H. Bloemen, ${ }^{* *}$ W. Collmar, ${ }^{*}$ R. Diehl, ${ }^{*}$ \\ W. Hermsen, ** G. Lichti, ${ }^{*}$ M. McConnell, *** J. Ryan,*** \\ V. Schönfelder, ${ }^{*}$ G. Stacy, ${ }^{* * *}$ A. W. Strong, ${ }^{*}$ \\ B.N. Swanenburg, ${ }^{* *}$ M. Varendorff* and O. R. Williams $\dagger$ \\ * Max-Planck-Institut für Extraterrestrische Physik, 8046 Garching, Germany \\ ** SRON-Leiden, P.O. Box 9504, 2300 RA Leidem. The Netherlands \\ *** University of New Hampshire, Durham, NH 03824, USA \\ † Astrophysics Division, European Space Research and Technology Centre, \\ Noordwijk, The Netherlands
}

\begin{abstract}
During the first part of the COMPTON Gamma Ray Observatory sky survey, COMPTEL has detected the quasars 3C273 and 3C279 and the radio galaxy Centaurus $\mathrm{A}$. This paper summarizes the preliminary findings and gives an upper limit on the MeV flux of the Seyfert galaxy NGC4151.
\end{abstract}

\section{INTRODUCTION}

Although extragalactic gamma-ray astronomy barely existed prior to the launch of the COMPTON Gamma Ray Observatory (CGRO), there were good indications that this is a potentially very promising field of research. Particularly good indicators were the detections of 3C273 by COS-B at energies above $50 \mathrm{MeV}$ $/ 1 /$ (suggesting a peak energy output in the $\mathrm{MeV}$ range) and the detections of Cen A /2/ and NGC 4151 $/ 3 /$ at $\mathrm{MeV}$ energies.

Since most of the COS-B observations were concentrated along the galactic plane, there was some hope that the GRO pointings away from the plane might show additional AGNs. This expectation has been borne out, since the EGRET experiment on CGRO (operating at energies $\gtrsim 50 \mathrm{MeV}$ ) has already detected more than a dozen AGNs.

The COMPTEL telescope aboard CGRO provides for the first time extensive imaging possibilities at MeV gamma-ray energies (about $0.75-30 \mathrm{MeV}$ ) with a source-location accuracy of typically $1^{\circ}$. A search for AGNs is underway; this paper reports on a few promising candidates, the radio galaxy Cen $\mathrm{A}$, the quasars $3 \mathrm{C} 273$ and 3C279 (both seen also by EGRET), and the Seyfert Galaxy NGC 4151.

\section{CENTAURUS A}

Cen $\mathrm{A}$ was in the field of view of COMPTEL several times during the sky survey (viewing periods $12,14,23$ and 27). When all four observations are combined, Cen $A$ is clearly seen at a flux level of about $1.2 \times 10^{-4}$ photons $\mathrm{cm}^{-2} \mathrm{~s}^{-1}$ in the energy range $0.75-1 \mathrm{MeV}$ (see Figure 1). Preliminary analyses of the individual observation periods do not show convincing detections of Cen A. Also shown in Figure 1 are COMPTEL $2 \sigma$ upper limits obtained from observation period 12 (Oct. 1991), together with the simultaneously measured OSSE spectrum, and other earlier measurements. It can be seen that the COMPTEL upper limits are at least an order of magnitude below the spectrum obtained with the MPI Compton Telescope $/ 2 /$.

\section{C273 AND 3C279}

The first observation in the direction of 3C273 (observation period 3, June 1991) surprisingly showed two sources of $\gamma$-rays in the wide field of view of COMPTEL and EGRET. Previously, COS-B had only detected 3C273. The second source was soon identified as another well known quasar, 3C279. As seen by EGRET at energies above $50 \mathrm{MeV}$, this object was very bright, much brighter, in fact, than $3 \mathrm{C} 273 / 5,6 /$. Meanwhile, at energies below $3 \mathrm{MeV}$ (as seen by COMPTEL), 3C273 was the dominating source.

Figure 2 shows the energy spectra for both sources as measured by COMPTEL and EGRET. In the COMPTEL energy domain their spectra appear to be distinctly different. For 3C273, the COMPTEL measurements 
are consistent with an $E^{-2.3}$ differential energy spectrum. The spectrum of 3C279 is much harder at COMPTEL energies, consistent with a power-law spectrum with index of about -1.3 to -1.6 . Both spectra connect smoothly to the EGRET measurements.

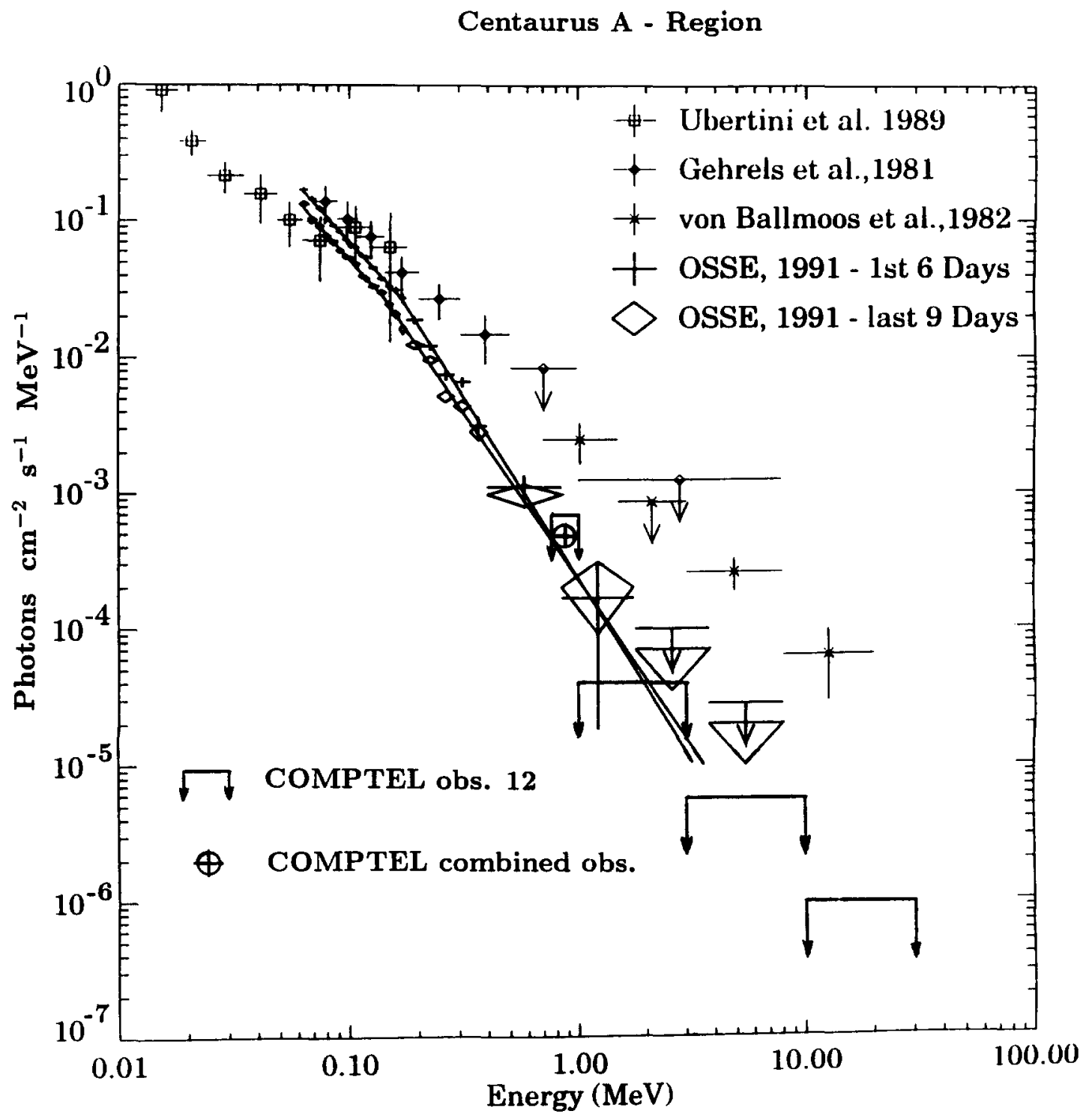

Fig. 1. Summary of Cen A measurements (from /4/, with COMPTEL results added). Note that only the COMPTEL upper limits $(2 \sigma)$ and the OSSE measurements were obtained simultaneously (observation period 12, Oct. 1991). The COMPTEL detection of Cen A (0.75-1 MeV) shown in this figure was obtained from a combination of four observations (see text).

Figure 3 shows 'luminosity' spectra of both sources in comparison with data from other instruments over a wide energy range (note that only the COMPTEL and EGRET observations are simultaneous). The luminosity distribution of $3 \mathrm{C} 273$ reaches a maximum around $10^{20} \mathrm{~Hz}(\sim 1 \mathrm{MeV})$. Above this energy the luminosity decreases. For 3C279 the maximum luminosity is reached around $3 \times 10^{21} \mathrm{~Hz}(\sim 10 \mathrm{MeV})$ where a change in the slope of the spectrum from spectral index -1.4 to -2.0 towards higher energies is observed. The difference between the spectra of $3 \mathrm{C} 273$ and $3 \mathrm{C} 279$ might be due to different emission processes in the two AGNs. At least the 3C279 spectrum cannot be genertated by $\gamma \gamma$ pair production because, in this case, the energy of the absorbed radiation must appear at lower energies in the spectrum, which is not seen. Thus the observed high-energy $\gamma$ radiation must originate in a region optically thin for $\gamma$-rays. 

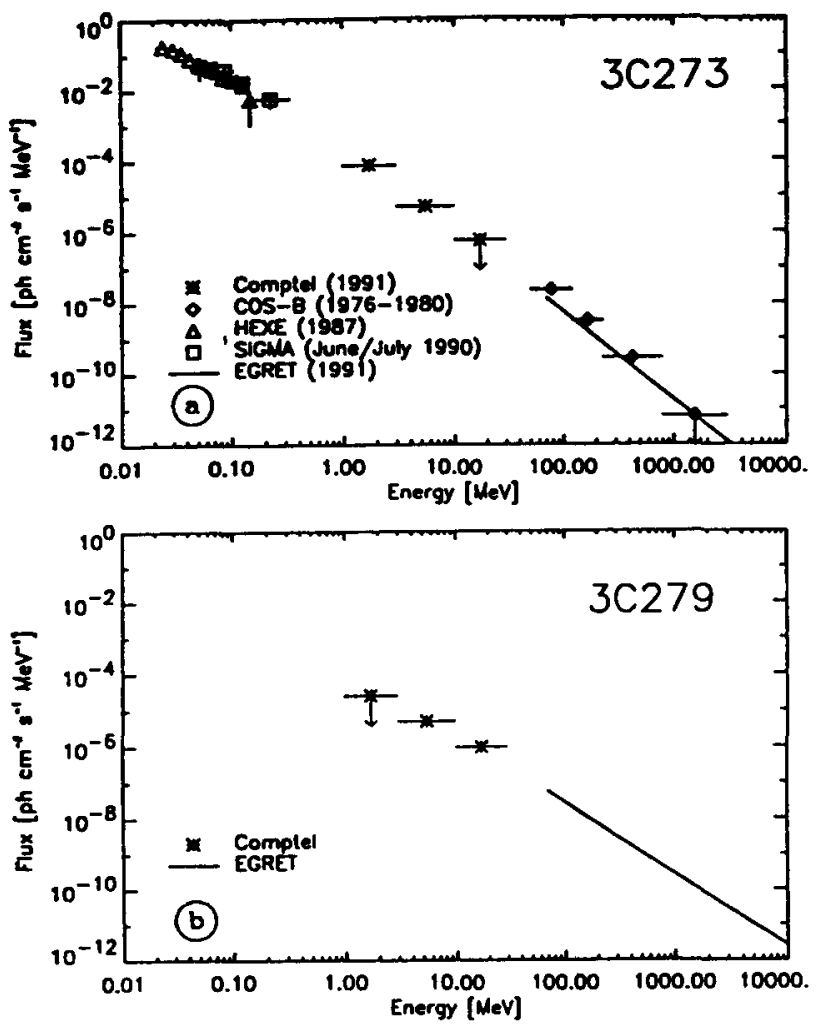

Fig. 2. Differential energy spectra: a) 3C273 - COMPTEL fluxes and spectra from COS-B, HEXE, SIGMA, and EGRET.

b) 3C279 - COMPTEL fluxes together with EGRET spectrum.

COMPTEL upper limits are $2 \sigma$.

Figure from $/ 7 /$; see there for references.

Following a "standard" model for AGNs, which assumes an accretion disk around a rotating black hole, the origin of the $\gamma$-rays cannot be close to the black hole as this region would be optically thick for $\gamma$-rays. Thus our observations favour models (e.g. $/ 8,9,10,11 /$ ) in which the $\gamma$-rays are produced further out, in or near

Fig. 3. 'Luminosity' spectra :

a) 3 C273 - COMPTEL data together with other spectra as in Figure 1a ;

b) 3C279 - COMPTEL and EGRET data together with measurements at lower frequencies for quiescent (open symbols) and enhanced (filled symbols) periods. X-ray data are from Ginga.

Figure from $/ 7 /$; see there for references.
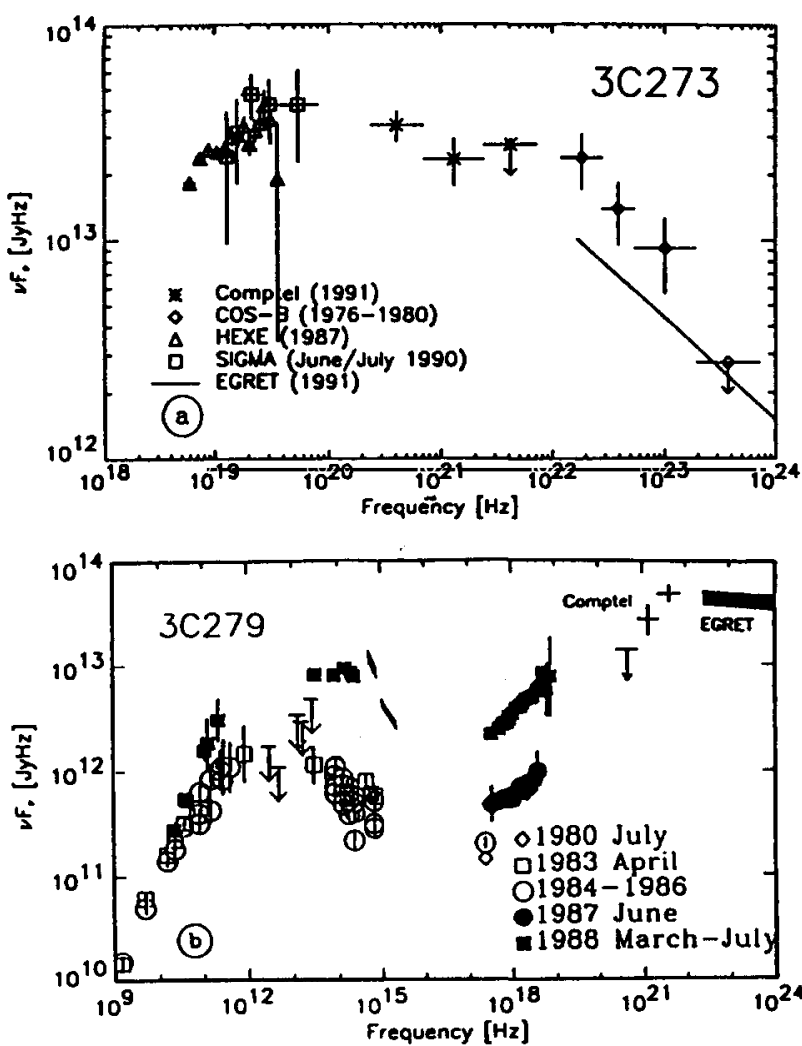
the jets ejected from the central engine. Inverse-Compton scattering of low-energy photons, or Doppler boosted electron-positron annihilation, in these beams might produce the $\gamma$-rays. In addition, because of the implied beaming effects, such models can reduce the total luminosity problem by several orders of magnitude $/ 12 /$.

\section{NGC 4151}

During observation period 4, the Seyfert galaxy NGC4151 was in the field of view of COMPTEL. A preliminary analysis showed no evidence of a detection. A conservative $3 \sigma$ upper limit on this source is $1.6 \times 10^{-4}$ photons $\mathrm{cm}^{-2} \mathrm{~s}^{-1}$ in the energy interval $1-30 \mathrm{MeV}$.

During the part of the sky survey completed sofar, many possible candidates of $\gamma$-ray emitting AGNs have been in the field of view of COMPTEL and EGRET. The detections of two AGNs and a radio galaxy during the far from completed analysis of the COMPTEL data and the detection of a quite large number of AGNs in the high-energy $\gamma$-ray range by EGRET are encouraging. We have therefore initiated a more detailed search for AGNs in the COMPTEL data, with an emphasis on those detected by EGRET and others considered as potential $\gamma$-ray sources.

\section{REFERENCES}

1. W. Hermsen, K. Bennett, G.F. Bigniami, J.B.G.M. Bloemen, R. Buccheri, P.A. Caraveo, G. Kanbach, J.L. Masnou, H.A. Mayer-Hasselwander, J.A. Paul, B. Sacco, R.D. Wills, Further COS-B evidence for Gamma-Ray emission from 3C273, Proc. 17th ICRC, 1, 230, (1981)

2. P. von Ballmoos, R. Diehl, and V. Schönfelder, Centaurus A Observation at MeV-Gamma-Ray Energies Astrophysical Journal, 312, 134-142 (1987)

3. F. Perotti, A. Della Ventura, G. Villa, G. Di Cocco, L. Bassani, R.C. Buttler, J.N. Carter, and A.J. Dean, Detection of a Soft Gamma-Ray Emission From the Region of NGC 4151, Astrophysical Journal, 247, L63-L66 (1981)

4. W.N. Johnson, J.D. Kurfess, W.R. Purcell, S.M. Matz, M.P. Ulmer, M.S. Strickman, R.J. Murphy, D.A. Grabelsky, R.L. Kinzer, G.H. Share, R.A. Cameron, R.A. Kroeger, M. Maisack, G.V. Jung, C.M. Jensen, D.D. Clayton, M.D. Leising, J.E. Grove and C.S. Dyer, Initial Results from OSSE on the Compton Observatory, Astronomy $\mathcal{B}$ Astrophysics, in press (1992)

5. C. von Montigny, D.L. Bertsch, C.E. Fichtel, R.C. Hartman, S.D. Hunter, G. Kanbach, D.A. Kniffen, P.W. Kwok, Y.C. Lin, J.R. Mattox, H.A. Mayer-Hasselwander, P.F. Michelson, P.L. Nolan, K. Pinkau, H. Rothermel, E. Schneid, M. Sommer, P. Sreekumar and D.J. Thompson, EGRET Observations of 3C273, Astronomy $\mathcal{B}$ Astrophysics, in press (1992)

6. R.C. Hartmann, D.L. Bertsch, C.E. Fichtel, S.D. Hunter, G. Kanbach, D.A. Kniffen, P.W. Kwok, Y.C. Lin, J.R. Mattox, H.A. Mayer-Hasselwander, P.F. Michelson, H.I. Nel, P.L. Nolan, K. Pinkau, H. Rothermel, E. Schneid, M. Sommer, P. Sreekumar, D.J. Thompson and C. von Montigny, Detection of High Energy Gamma Radiation From Quasar 3C279 by the Gamma Ray Observatory, Astrophysical Journal, 385, L1 (1992)

7. W. Hermsen, H.J.M. Aarts, K. Bennett, H. Bloemen, H. de Boer, W. Collmar, A. Connors, R. Diehl, R. van Dijk, J.W. den Herder, L. Kuiper, G.G. Lichti, J.A. Lockwood, J. Macri, M. McConnell, D. Morris, J.M. Ryan, V. Schönfelder, G. Simpson, H. Steinle, A.W. Strong, B.N. Swanenburg, C. de Vries, W.R. Webber, O.R. Williams, C. Winkler, COMPTEL detections of the quasars 3C273 and 3C279, Astronomy $\&$ Astrophysics, in press (1992)

8. C.D. Dermer, R. Schlickeiser, and A. Mastichiadis, High-energy gamma radiation from extragalactic radio sources, Astronomy $\&$ Astrophysics, 256, L27-L30 (1992)

9. K. Mannheim and P.L. Biermann, Gamma-ray flaring of 3C279: a proton-initated cascade in the jet? Astronomy \& Astrophysics, 253, L21-L24 (1992)

10. L. Maraschi, G. Ghisellini, and A. Celotti, A Jet Model for the Gamma-Ray-Emitting Blazar 3 C279 Astrophysical Journal, 397, L5-L9 (1992)

11. G. Henri, G. Pelletier, and J. Roland, Gamma Ray Emission of AGNs as a Signature of Relativistic Electron-Positron Beams, Astrophysical Journal, in press (1992)

12. M. Camenzind, M. Krockenberger, The lighthouse effect of relativistic jets in blazars, Astronomy $\mathscr{B}$ Astrophysics, 255, 59-62 (1992) 